\title{
Preparation of Methylphenylvinyl Raw Rubber by Bulk Polymerization and Ring-Opening Polymerization Methods
}

\author{
Ting LIU ${ }^{\mathrm{a}}$, Shi-Jian CHEN ${ }^{\mathrm{b}}$, Bo-Quan JIANG ${ }^{*}$ \\ Department of Biology and Chemistry, Institute of Science, Nanchang University College of Science \\ and Technology, Nanchang, Jiangxi, 330029,China. \\ apartrickheroin@sohu.com, bcsi440@qq.com, jjbq_win@163.com \\ ${ }^{*}$ Corresponding author
}

Keywords: Methylphenylvinyl Raw Rubber, Bulk Polymerization, Ring-opening Polymerization, Methylphenolcyclosilicone, Methylphenyldiethoxsilane.

\begin{abstract}
Methylphenylvinyl raw rubber (MPVRR) is one of the important materials for preparation of phenyl-containing additive molding liquid silicone rubber (AMLSR). Two methods, bulk polymerization (BP) and ring-opening polymerization (ROP), were used to prepare MPVRR. The effects of formulation, temperature and water content on MPVRR properties for BP method were investigated and the product was characterized by Fourier Infrared Spectrometer. The effects of formulation, end capping reagent dosage, feeding sequence, reaction temperature and time on MPVRR properties for ROP method were discussed and the product was characterized by Double Beam UV-visible Spectrophotometer. The results showed that BP method could only prepare low phenyl content of MPVRR with phenyl mass percentage of 3.4\%-5.2\%, but ROP method could prepare both low phenyl content of MPVRR with phenyl mass percentage of $9.2 \%$ and middle phenyl content of MPVRR with phenyl mass percentage of $16.4 \%$, which were nearly close to the theoretical values of $12 \%$ and $21.5 \%$, respectively. The samples prepared using the two methods were proved to be MPVRR materials by the instrument analysis, respectively.
\end{abstract}

\section{Introduction}

Methylphenylvinyl raw rubber (MPVRR), also called as vinyl-terminated polymethylphenylsiloxane, is one of the main components of additive molding liquid silicone rubber (AMLSR). The AMLSR consists of MPVRR, hydrogen silicone, oil-containing crosslinking agent, catalyst, etc. High quality MPVRR is needed to meet the requirement of AMLSR preparation because the vulcanization of ethylene with silicon hydrogen bases depends on the molar ratio of ethylene/silicon hydrogen base in ACLSR preparation. If some byproducts are formed in MPVRR preparation, it will affect the performance of AMLSR.The synthesis of phenyl-containing silicone polymer is similar to that of normal organic silicon polymer. The methods include bulk polymerization, solvent polymerization, emulsion polymerization, suspension polymerization and ring-opening polymerization, among which, bulk polymerization (BP) and ring-opening polymerization(ROP) methods are usually used. BP, generally consisting of monomer synthesis, separation and hydrolysis, de-polymerization and ring opening polymerization of hydrolysate, can be directly conducted by the action of benzene-containing silane and ring siloxane with the help of end capping reagent, catalyst, chain transfer agent and stabilizer. BP method has some advantages such as simple technique, short process, less equipment, low investment and easy operation. Ring-opening polymerization is a process in which the cyclic monomers are opened to form linear polymers under the action of initiator or catalyst. The characteristics of ROP are different from addition polymerization and polycondensation, but similar to them in some aspects. The catalysts used for ROP are divided into the alkaline and acidic. The ROP with acidic catalyst or alkaline catalyst are called cationic ROP or anionic ROP, respectively. Under 
most conditions, the anionic ROP method is used due to its more convenience and effectiveness than cationic ROP. The cationic ROP is only used when alkaline catalyst cannot be used for the polymerization. So far, only a few of articles reported the preparation of hydroxyl-terminated poly methyl phenyl siloxane [1-2], however, no reports on the preparation of vinyl-terminated poly methyl phenylsiloxane by BP and ROP methods were found in literature. In this paper, the two methods above were used to prepare MPVRR and compared in operation procedure, technical parameters and product properties.

\section{Experimental}

\section{Preparation of MPVRR by BP Method}

Preparation of Alkali Rubber. Alkali rubber (tetramethyl ammonium hydroxide silicon alkoxide, TAHSA) is the catalyst for the preparation of MPVRR. The alkali rubber was prepared by following procedure.

$1 \mathrm{~g}$ of $50 \%$ tetramethyl ammonium hydroxide was added into $50 \mathrm{~g}$ D4 in a distillation unit. After dry nitrogen passed into the unit for a short time,the unit was vacuumed to $1.33 \mathrm{KPa}$ with stirring. The mixture was slowly heated up to $40{ }^{\circ} \mathrm{C}$ and maintained the same temperature for $0.5 \mathrm{~h}$ for dehydration and then continued to be heated up to $80{ }^{\circ} \mathrm{C}$ at $13.33 \mathrm{KPa}$, while water was constantly steamed out and the mixture viscosity gradually increased. Afterwords, the pressure was decreased to $1.33 \mathrm{KPa}$ again until obtaining a translucent dope and then sealed and stored the dope for use[3]. During the catalytic polymerization reaction, the $-\mathrm{SiO}$ bonds strongly attacked the - SiOSi bonds in D4 to make the siloxane chains gradually grow.

Preparation of MPVRR. (1) Method. The monomer MPDS, double head $\mathrm{MM}^{\mathrm{Vi}}$, dimethyl formamide(DMF) and TAHSA(self-made) were taken as phenyl source, end capping reagent, promoter and catalyst, respectively. The polycondensation reaction between MPDS and hydroxyl end polysiloxane HODnH, obtained by the ring-opening polymerization of $\mathrm{D} 4$, took place to prepare MPVRR. The reaction formula is as follows.

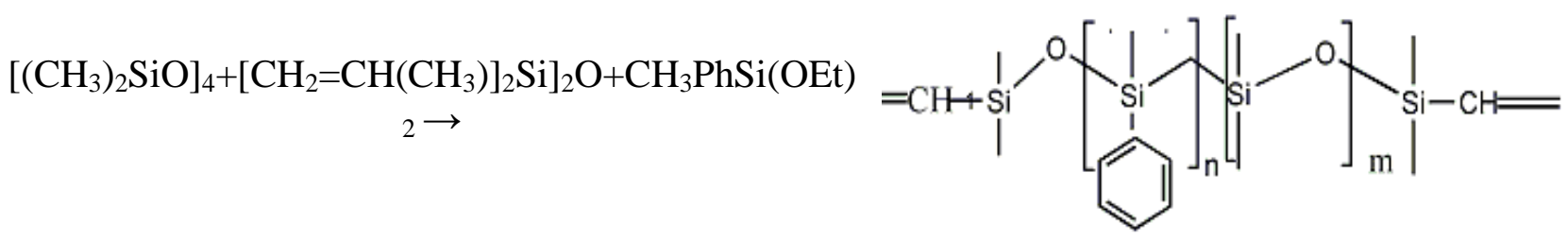

(2) Procedure. The procedure was conducted as follows: (i) adding certain amounts of D4, DMF and MPDS, which were calculated according to the reaction formula, into the reactor and then heating the reactants with stirring; (ii) gradually passing the dry nitrogen into the reactor until the temperature up to $90^{\circ} \mathrm{C}$, while accelerating the flow rate of nitrogen gas until the water water was removed as completely as possible;(iii) adding the catalyst into the reactor and maintaining the reaction temperature of about $110^{\circ} \mathrm{C}$ (iv) adding the end capping reagent into the reactor when the mixture became transparent thick shape and keeping the reaction until no obvious viscosity change of the product; (v) rapidly increasing the temperature up to $180-190{ }^{\circ} \mathrm{C}$ and then adding a large number of dry nitrogen for removing the alkali rubber catalyst;(vi)after about two hours, increasing the temperature up to $200^{\circ} \mathrm{C}$ under decompression and removing the components with low boiling points to obtain the colorless and transparent product(MPVRR).

\section{Preparation of MPVRR by ROP Method}

Method. The ring-opening polymerization reaction was carried out between the MPDS' hydrolysate (MPCS) and D4 with double head MMVi, dimethyl formamide and tetramethyl ammonium hydroxide 
(TMAH) as end capping agent, promoter and catalyst, respectively. The reaction formula is as follows:

$\left.\left[\left(\mathrm{CH}_{3}\right)_{2} \mathrm{SiO}\right]_{4}+\left[\mathrm{CH}_{2}=\mathrm{CH}\left(\mathrm{CH}_{3}\right)\right]_{2} \mathrm{Si}\right]_{2} \mathrm{O}+\left(\mathrm{CH}_{3} \mathrm{PhSiO}\right) \mathrm{n}$

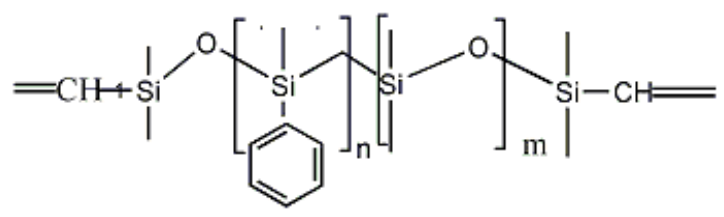

Where $\left.\mathrm{CH}_{3} \mathrm{PhSiO}\right) \mathrm{n}(\mathrm{n} \geq 3)$ is the methylphenylcyclosiloxane mixture.

Preparation Procedure. The procedure was conducted as follows: (i) adding certain amounts of D4, DMF and MPCS, calculated according to the reaction formula, into the reactor and then heating the reactants with stirring; (ii) gradually passing the dry nitrogen into the reactor until the temperature up to $90^{\circ} \mathrm{C}$, while increasing the flow rate of dry nitrogen gas in order to remove the water as completely as possible, then stopping nitrogen addition;(iii) adding the tetramethyl ammonium hydroxide catalyst into the reactor and maintaining the reaction temperature of about $110^{\circ} \mathrm{C}$ (iv) adding the end capping reagent into the reactor when the reaction mixture changed from white to pale yellow color and transparent thick shape, and then controlling the reaction time for $4 \mathrm{~h}$; (v) after rapidly increasing the temperature up to $180-190{ }^{\circ} \mathrm{C}$ and adding a large number of dry nitrogen for removing the catalyst;(vi)after about two hours, increasing the temperature up to $200{ }^{\circ} \mathrm{C}$ under decompression and removing the components with low boiling points to obtain the colorless and transparent product(MPVRR).

Phenyl Content Measurement. The ultraviolet absorption spectroscopy method was used to measure the phenyl content of MPVRR. The product absorbance was determined by double beam uv-visible spectrophotometer (T-1901type, China). The phenyl molar content was calculated according to the standard curve[4].

\section{Results and Discussions}

\section{MPVRR Preparation by BP Method}

Formulations and Product Properties. Two formulations for MPVRR preparation and their product properties are compared as listed in Tab.1.

Tab.1 Formulations for MPVRR preparation and their product properties

\begin{tabular}{cccccccc}
\hline No. & $\begin{array}{c}\text { Formulation } \\
\mathrm{MM}^{\mathrm{Vi}} \\
\end{array}$ & $\begin{array}{c}\text { MPDS } \\
/ \mathrm{g}\end{array}$ & $/ \mathrm{g}$ & $\begin{array}{c}\text { TAHSA } \\
/ \mathrm{g}\end{array}$ & $\begin{array}{c}\text { maximum absorbance } \\
(\text { near } 264 \mathrm{~nm})\end{array}$ & $\begin{array}{c}\text { refractive index } \\
\left(30^{\circ} \mathrm{C}\right)\end{array}$ & $\begin{array}{c}\text { phenyl content } \\
/ \%\end{array}$ \\
\hline & & & & & & & \\
1 & 25 & 8.8 & 0.2 & 0.24 & 0.106 & 1.4112 & 3.4 \\
2 & 25 & 20 & 0.2 & 0.24 & 0.151 & 1.4153 & 5.2
\end{tabular}

It can be seen from Table1 that the phenyl contents obtained by formulation 1 and formulation 2 are $3.4 \%$ and $5.2 \%$, respectively, which are much lower than those of theoretical values of $11 \%$ and $22 \%$ due to less phenyl accessed to the main chains of the product, indicating that only low phenyl level MPVRR could be prepared by the bulk polymerization of MPDS with D4.

Effect of Temperature. It was found in the experiment that D4 was easily polymerized with MPDS by alkali catalyst at $60^{\circ} \mathrm{C}$. So, the temperature should be strictly controlled not to be too high when D4 and MPDS were heated and dehydrated under decompression and low temperature before reaction, otherwise, the reactants would begin to polymerize before incomplete dehydration, leading to the reaction of water with D4 by alkali rubber catalyst, to form silicon hydroxyl, which will seriously 
influence the product quality. So, the temperature should be increased to begin the polymerization after dehydrating for a half an hour.

Effect of Water Content. During the preparation of alkali rubber, a small amount of water would affect the activity of the catalyst due to very low catalyst concentration in the system. At the same time, if there exists water in the system, the hydrolysis of polysiloxane would happen under alkali catalysis to generate hydroxyl silicon. If the product contains hydroxyl silicon groups, they will react with silicon hydrogen bases contained in the crosslinking agents during the vulcanization of silicone rubber, which will result in a decrease of the actual number of hydroxyl silicon groups reacted with silicon vinyl, and influence the performance of vulcanized rubber. Therefore, like alkali rubber preparation, the reactor should be dried and D4 should be dehydrated under depression before reaction and the dry nitrogen should be passed into the reactor in order to prevent the moisture contained in air into the reaction system. It was found in the experiment that when D4 contained more moisture before the reaction, the color of the formed rubber became dark and a poor quality product was obtained.

Infrared Spectra of MPVRR. The infrared spectra of MPVRR by Fourier infrared spectrometer (Nicolet 5700 type,US)was shown in Fig.1. From the figure we can see that the C-H peak at 2969 $\mathrm{cm}-1$ and two $\mathrm{C}-\mathrm{H}$ stretching absorption peaks in $-\mathrm{CH}=\mathrm{CH} 2$ vinyl group associated with $=\mathrm{CH} 2$ stretches at $3050 \mathrm{~cm}-1$ and $3072 \mathrm{~cm}-1$ indicate the $-\mathrm{CH}=\mathrm{CH} 2$ groups have been connected in the material;the peaks at $800.4 \mathrm{~cm}-1,1261 \mathrm{~cm}-1$ and $1390 \mathrm{~cm}-1$ are the characteristic peaks of silicon methyl(Si-CH3); the peak at $1081 \mathrm{~cm}-1$ is the characteristic peak of siloxane ( $\mathrm{Si}-\mathrm{O}-\mathrm{Si})$ and the peaks at $1592 \mathrm{~cm}-1,1429 \mathrm{~cm}-1,737 \mathrm{~cm}-1$ and $700 \mathrm{~cm}-1$ are the characteristic peaks of silicon phenyl (Si-C6H5).These characteristic peaks proved that the sample prepared in the experiment is vinyl-terminated polymethylphenylsiloxane, which are similar to those in literature [5].

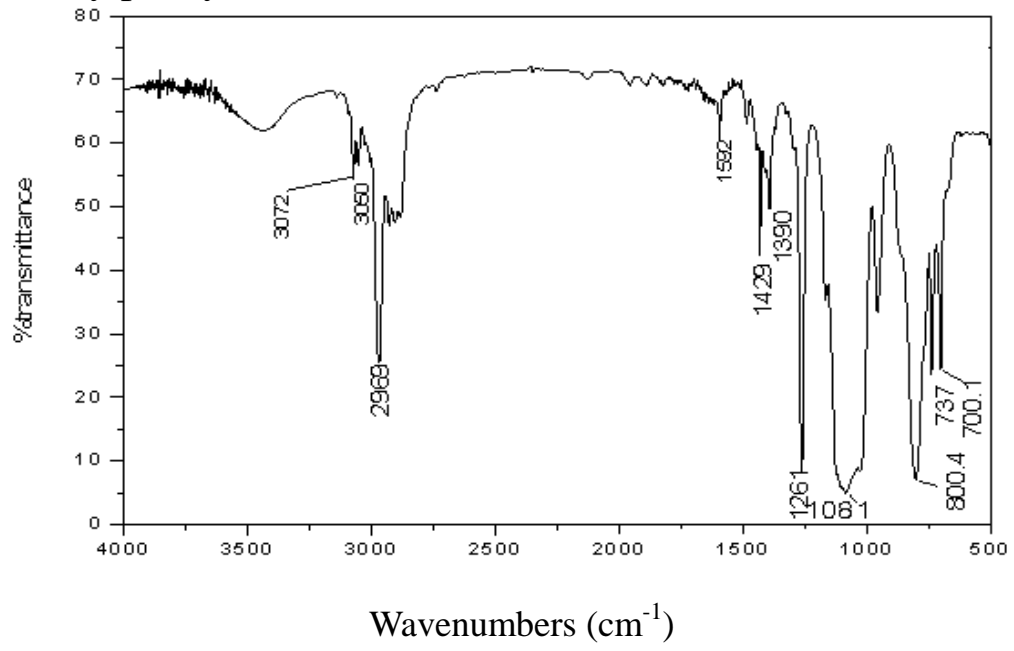

Fig.1 FTIR spectra of MPVRR)

\section{MPVRR Preparation by ROP Method}

Formulations and Product Properties. Three formulations for MPVRR preparation and their product properties are compared as listed in Tab.2.

It can be seen from Tab.2 that three formulations obtain three kind of MPVRRs with different phenyl contents of $0 \%, 9.2 \%$ and $16.4 \%$, represented with $\mathrm{i}$, ii and iii respectively. The three products are called zero phenyl MPVRR, low phenyl MPVRR( phenyl content ranged from 5\% to 10\%) and middle phenyl (phenyl content ranged from $10 \%$ to 20\%), respectively. The UV-spectrogram of low phenyl MPVRR (ii) and middle phenyl MPVRR (iii) are shown in Fig.2 and Fig.3, respectively. It can be known from Table2 and Fig.2-3, that the phenyl content increases with increasing MPCS dosage and the phenyl content in MPVRR ii (9.2\%)and MPVRR iii (16.4\%) are close to the theoretical values of 
$12 \%$ and $21.5 \%$, respectively. However, too high MPCS dosage leads a great drop of phenyl number due to the poor compatibility of MPCS with D4, uneven dispersion of MPCS and larger viscosity of the reaction mixture with increasing phenyl content, resulting in a reduction of phenyl ring body conversion.

Tab.2 Formulations for MPVRR preparation and their product properties

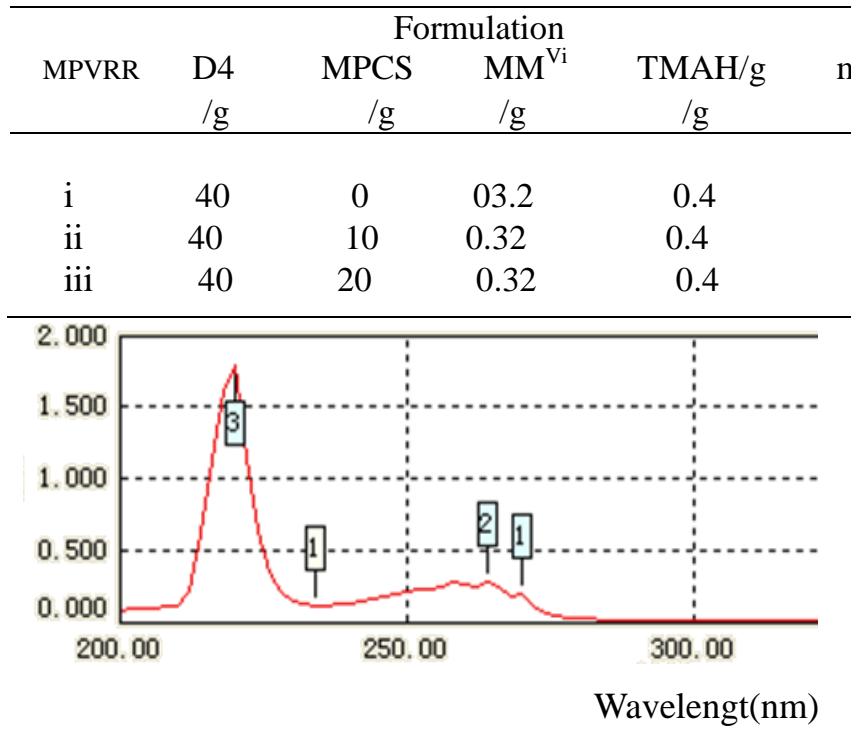

Fig.2. UV-spectrogram of MPVRR (ii)

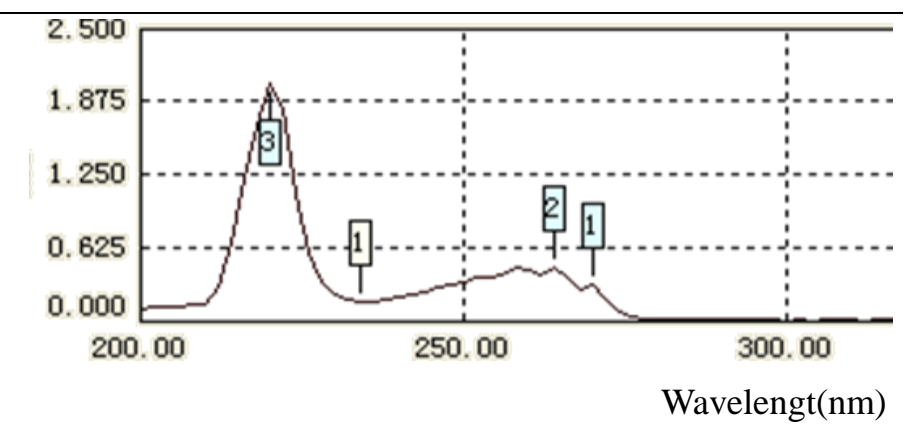

Fig.3. UV-spectrogram of MPVRR (iii)

Effect of End Capping Reagent. The end capping reagent (MMVi) is used as vinyl source of MPVRR and its dosage determines the viscosity of MPVRR when the other conditions are fixed. Tab.3 shows the effect of end capping reagent dosage on the viscosity of MPVRR. It can be seen from Table 3 that the viscosity of MPVRR increases with decrease of MMVi dosage and is greatly increased when the mass ration of $\mathrm{MMVi} / \mathrm{D} 4$ is decreased to less than 0.6, which is in agreement with that reported in literature [6]. It is also found that the viscosity of MPVRR increases with increasing phenyl content in MPVRR under the same MMVi dosage.

Tab.3. Effect of end capping reagent dosage on viscosity of MPVRR

\begin{tabular}{ccccc}
\hline Mass ratio of MMVi /D4, \% & 0.8 & 0.7 & 0.6 & 0.5 \\
\hline $\begin{array}{c}\text { Viscosity of MPVRR(i), } \\
\left(20^{\circ} \mathrm{C}\right) / \text { mpa.s }\end{array}$ & 1510 & 1920 & 2735 & 4110 \\
$\begin{array}{c}\text { Viscosity of MPVRR (ii) } \\
\left(20^{\circ} \mathrm{C}\right) / \text { mpa.s }\end{array}$ & 1625 & 2080 & 2850 & 4235 \\
$\begin{array}{c}\text { Viscosity of MPVRR (iii) } \\
\left(20^{\circ} \mathrm{C}\right) / \text { mpa.s }\end{array}$ & 1820 & 2280 & 2955 & 4450 \\
\hline
\end{tabular}

Effect of Feeding Sequence. It was found during the experiment that when the end capping reagent was added into the polymerization system before adding catalyst, the activity of catalyst would be reduced which affected the reaction. Therefore, we firstly added the catalyst into the hybrid ring bodies, and then added the end capping reagent into the system when the mixture became transparent with high viscosity, by which the appearance of the prepared product could meet the index requirements of MPVRR. 
Effect of Reaction Temperature and Time. In the ring-opening polymerization, too low temperature will slow down ring-opening reaction and too high temperature will lead to the decomposition and activity decrease of the catalyst. So, it is important to determine the suitable temperature in the polymerization process. We investigated the effect of temperature on the polymerization and the results showed that an ideal polymerization reaction could be conducted at $90-110{ }^{\circ} \mathrm{C}$, otherwise, the catalyst began to decompose and deactivate when the temperature was above $130{ }^{\circ} \mathrm{C}$. Reaction time is also an important factor to depend on the MPVRR quality. By the investigation, the appearance of the prepared MPVRR could meet the requirements of MPVRR when the reaction time was controlled within 5-8 h.

\section{Conclusions}

MPVRR was successfully prepared by BP method with MPDS as raw material and ROP method with MPCS as raw material, respectively.By the comparison of the two methods, the BP method could prepare low phenyl MPVRR with phenyl content of 3.4\% to 5.2\% and the ROP method could prepare low phenyl MPVRR and middle phenyl MPVRR with phenyl contents of 9.2\% and 16.4\%, respectively, which were nearly close to the theoretical values of $12 \%$ and $21.5 \%$, respectively. The optimal conditions for preparing MPVRR by ROP method were determined to be: reaction temperature of $110^{\circ} \mathrm{C}$, reaction time of $6 \mathrm{~h}$. The end capping reagent was suggested to be added into the polymerization system after catalyst and the mass ratio of MMVi /D4 should be controlled to prepare MPVRR with suitable viscosity. The following work will focus on the preparation of additive molding liquid silicone rubber with MPVRR as raw material, prepared in the experiment.

\section{References}

[1] Meijiang Li, Mingjun Wu, Jianjin Wu,et al. Chemical Production and Technology 18(2011),P.21.

[2]Cheng Yang, Hualan Wang,Jirong Wu, et al. Journal of Hangzhou Nomal University(natural science edition) 13(2014),P. 6.

[3]Zhongwen Liu. Organic Silicon Materials and Applications1(1999),P. 3.

[4]Zhiying Li. Industrial analysis of organic silicon compound.[M].Beijing:Chemical industry press, 1979,Beijing, China.

[5]Mengxian Luo, Meijiang Li, Yan Wang, et.al. Silicone Material, 22(2008),p.335.

[6]Songmin Xing, Yilu Wang. Organic silicon synthesis technology and product application[M]. Beijing:Chemical industry press, 2000, Beijing, China. 